Notre Dame Journal of Formal Logic

Volume VIII, Numbers 1 and 2, April 1967

In memoriam

WILHELM ACKERMANN

1896-1962

\title{
HANS HERMES
}

Wilhelm Ackermann was born on March 29, 1896, in Schönebeck (Kreis Altena) in Westphalia, at that time belonging to Prussia. His studies at the University of Göttingen, which were interrupted during the First World War, were devoted to mathematics, physics, and philosophy. He obtained his Ph.D. in 1924 as a student of David Hilbert. Then a scholarship enabled him to spend time in Cambridge (England). From 1927 until 1961 he taught in secondary schools, first in Burgsteinfurt (Westphalia), then as an Oberstudienrat in Lüdenscheid (Westphalia). In 1953 he became a corresponding member of the Göttingen Academy of Sciences, and in the same year the School of Mathematics and Sciences (Mathematisch Naturwissenschaftliche Fakultät) of Münster University made him an honorary professor. While continuing his teaching position in Lüdenscheid, he gave regular lectures at the University on questions of mathematical logic and the foundations of mathematics. He lectured until three days before his death on December 24, 1962.

Although Ackermann did not choose a university career, he was continually engaged in research and published many contributions to the foundations of mathematics. He became especially well known as the main author of the textbook: Hilbert-Ackermann Grundzüge der theoretischen Logik [4]. Between 1928 and 1959 this book has had four editions and has been translated into several languages. Being a student of Hilbert, Ackermann began with investigations on the consistency of mathematics. The mathematical systems which he investigated were at first of a rather limited kind. But later he also studied more comprehensive systems of set theory and logic without types. His last papers contain sketches of systems which seem to be consistent, but the consistency of which has not been proven yet. Besides this main branch of investigations, Ackermann has, in several publications, been concerned with the decision problem of predicate logic. In 1954 a comprehensive presentation was published in book form under the title Solvable Cases of the Decision Problem [19]. Finally, there are some shorter investigations concerning recursive functions and the strengthening of strict implication. 
1. Investigations Concerning the Consistency of Arithmetic. In his famous paper for the International Congress of Mathematicians in 1900, Hilbert had mentioned as the second problem the task of proving the consistency of mathematics. Thereby, Hilbert wanted to overcome the difficulties which had arisen because of the antinomies in set theory. A proof theory should investigate mathematical proofs. With finitistic arguments it should be established that the usual forms of proof of traditional mathematics, including the tertium non datur, could not lead to contradictions. The realization of Hilbert's program waited a long time. The first major contribution was the 1924 dissertation [1] of Ackermann in which the consistency of a part of the arithmetic of natural numbers was proved. The formal system investigated by Ackermann is based on the classical logic of propositions and predicates with identity. To these are added the axioms of Peano for arithmetic and additional axioms which allow the introduction of new functions by various recursive procedures.

The main idea of the proof of consistency given by Ackermann is the following: arithmetic of natural numbers as described above can be constructed in such a way that, besides the rule of substitution, the rule of detachment (i.e. modus ponens which in Hilbert's terminology is simply called the schema of derivation) is the only rule of derivation. By repeating in certain cases some parts of the proof, every proof can always be represented in the form of a tree. The endings of the branches of such a tree are the axioms (which in general have to appear more than once). The stem is the proved theorem. From every branch ending exactly one way leads to the stem. Such a path is called a branch of the proof.

In order to show consistency one has to establish that there is no proof which ends with the formula $O \neq O$. Let us assume that there were such a proof. By a rather complicated procedure dealing with the tree of the proof, we can transform this proof step by step into new proofs which all end with the same formula $O \neq O$. Thereby it is possible to eliminate successively all free and bound variables. Finally, we get a proof for $O \neq O$ which contains no more variables which means that all the lines of it are numerical formulas. But for numerical formulas it is not difficult to introduce the concepts correct and false. It is easy to see that a proof which consists only of numerical formulas can contain only numerical formulas which are correct. Such a proof can therefore not end with the false formula $O \neq O$. This establishes the consistency of the formal system under consideration. An addition to this method is contained in the paper [2] from 1925. Here, fuller axioms are introduced, namely

$$
\hat{x}_{x}(A(x) \leftrightarrow B(x)) \rightarrow \varepsilon_{x}^{\varepsilon} A(x)={\underset{x}{\varepsilon}}_{B} B(x)
$$

and another similar axiom. By these axioms the role of the $\varepsilon$-operator as a selection operator (Auswahloperator) is defined.

Unfortunately, it turned out that the proof of Ackermann falls short of the end for which it was designed. It does not establish the consistency of the system of arithmetic (of natural numbers). Some applications of the schema of induction are not covered by Ackermann's proof. This fact is clearly brought out in the presentation of Ackermann's proof in $\$ 2$ of 
volume 2 of Hilbert and Bernays' Grundlagen der Mathematik published in 1939. A proof of consistency for the system of arithmetic (of natural numbers) was given by Gentzen in 1936. Later in 1940 in [13] Ackermann also gave such a proof.

At the very time at which the first effective proofs of consistency were constructed, Hilbert's proof theory underwent a serious crisis. In 1931 Gödel proved that the consistency of a calculus of arithmetic cannot be proved with the means of this calculus itself. Before, it had always been assumed that the finitistic means, with the help of which the consistency of a calculus could be proven, constituted but a proper part of the logical possibilities of the calculus in question. . This tacit assumption had to be dropped. But it was discovered that there could be finitistic methods of deduction which were not contained e.g. in the calculus of arithmetic. Such methods of derivation could be used in the proof of the consistency of the arithmetic of natural numbers. Thus it was possible to save the program of proof theory in spite of Gödel's theorem.

In 1937 [10] Ackermann reduced the consistency of a part of the axioms of set theory to the consistency of arithmetic of natural numbers. As his set theoretical axiom system he chose the system of Zermelo-Fraenkel including the axiom of choice and the axiom of subsets, but without the axiom of infinity and the axiom of replacement. The proof proceeds by introducing into number theory a two-placed predicate Exy, which means that the binary representation of $y$ contains a 1 at the $x$-th place. Ackermann shows that for this predicate (in place of $\varepsilon$ ) the set theoretical axioms in question can be derived by means of the arithmetic of natural numbers. Thus the consistency of this part of set theory is reduced to the consistency of arithmetic of natural numbers.

In the same year in (11) Ackermann constructed a logic on a set theoretical basis. The system has certain affinity with a paper by Quine (The Journal of Symbolic Logic, vol. 1). Ackermann uses the Hilbert operator $\varepsilon$. Identity is introduced with the help of extensionality. If a formula $A(x)$ is given, then a term can be associated with it according to the following definition:

$$
\hat{x} A(x)=\underset{x}{\varepsilon} \hat{y}(x y \leftrightarrow A(y))
$$

With the help of this operator the notion of set is explained by

$$
M x \leftrightarrow \hat{y}(x y)=x
$$

By means of the language indicated Ackermann formulates the axioms of set theory. Because of the properties of the $\varepsilon$-operator the axiom of choice is derivable. Through a modification of the proof in [10], it is possible to prove also the consistency of the axiom system considered in [11] (however, without the axiom of infinity).

2. Investigations Concerning the Consistency of Comprehensive Mathematical Systems. The arithmetic of natural numbers is a relatively limited mathematical formal system. To continue the program of Hilbert the 
consistency of richer formal systems had to be investigated. Since 1900 different systems have been proposed. Several turned out to be inconsistent. In 1958 in the introduction of [24] ( $c f$. also the introduction of [27]) Ackermann, following Curry, gives a survey of the principles which have led to the construction of comprehensive formal systems. The authors of such systems have endeavored to realize certain general ideas. Thus they have arrived at e.g. the following postulates: (i) Every 'property' leads to the formulation of a predicate (set), (ii) The Aristotelian principle of bivalence is valid in the sense that for every predicate and for every thing it is meaningful to say that the predicate applies or does not apply to the thing, (iii) The theorem of deduction is valid for the system i.e., there is a truth functional connection $\rightarrow$, such that $A \rightarrow B$ is provable if $B$ is derivable from $A$. In no consistent formal system can all these ideas be realized at once. Ackermann distinguished systems of type I, where the consistency should be guaranteed by a limitation of (i), while the classical logic of propositions remains valid, and systems of type $\Pi$, where (i) is realized, but the classical logic of propositions is abandoned. To type I belong Russell's theory of types and the usual axiomatic systems of set theory. Systems of type II (or suggestions for their construction) are due to Behmann, Church, Schutte and Ackermann himself. [14], [15], [17], [18] , [24], [25] and [28] are concerned with the development of systems of type II and with the proof of consistency of such systems. The presentations in general are rather sketchy. [27] contains a survey of the development of systems of type II.

In 1956 in [20] Ackermann gives an axiom system for set theory which, by means of a suitable interpretation of Cantor's naive definition of a set, limits the formation of sets in a 'natural way'. Ackermann's system is characterized by the fact that in addition to $\varepsilon$ it contains a further primitive constant ' $M$ ' ('is a set' (Menge)). For the formation of 'things' and 'sets' , Ackermann gives the following axiom schemata:

$$
\begin{aligned}
& \text { (i) } \underset{x}{\wedge}(A(x) \rightarrow M x) \rightarrow \underset{y}{\vee} \underset{z}{\wedge}(z \varepsilon y \leftrightarrow A(z)) \\
& \text { (ii) } M x_{1} \wedge \ldots \wedge M x_{n} \wedge \wedge_{y}^{\wedge}\left(A_{0}(y) \rightarrow M y\right) \rightarrow \underset{z}{\vee}\left(M z \wedge \wedge \underset{u}{\wedge}\left(u \varepsilon z \leftrightarrow A_{0}(u)\right)\right.
\end{aligned}
$$

In (ii) the expression $A_{0}$ may not contain the $\operatorname{sign} M . x_{1}, \ldots, x_{n}$ are the free individual variables which occur in $A_{0}$ other than $y$.

A first attempt to construct a consistent system of type II was made by Ackermann in 1941 in [14] inspired by Behmann and Church. Ackermann had planned to prove in a second paper the consistency of his system. But this proof never appeared. Instead, in [24] Ackermann has made the conjecture that [14] might be inconsistent since it might give rise to an antinimony similar to the one which Kleene and Rosser have discovered in a system proposed by Church.

In a series of papers [15]-[18] from 1950-1953 Ackermann made a new start. In [15] a system is based on implication $\rightarrow$, which is introduced axiomatically and the consistency of this system is proved. In [17] a new variation of the construction in [15] is presented. The proof of consistency 
makes use of a system of constructive ordinal numbers that had been prepared in [16]. In [18] it is shown that in system [17] the arithmetic of natural numbers and a certain part of analysis can be constructed. Thus a result is achieved which is comparable to the proofs of consistency of Lorenzen (1951) and of Schütte (1954). The guiding principle of Ackermann's construction is the idea to interpret the implication $A \rightarrow B$ as ' $B$ is derivable from $A$ '. This does not immediately lead to the definition of implication, since into the definition of the notion of derivation enters modus ponens and thereby the implication itself. Instead, Ackermann tries to determine the implication axiomatically. With the above mentioned interpretation of implication in mind, one might take offense at a formula like $A \rightarrow(B \rightarrow A)$, which on the left contains a formula $A$ and on the right side a 'metaformula' $B \rightarrow A . \quad A \rightarrow(B \rightarrow A)$ is therefore replaced by $\left(C_{0} \rightarrow A\right) \rightarrow$ $(B \rightarrow A)$, where $C_{0}$ is a fixed provable formula.

3. A Strengthening of Strict Implication. In 1956-1958 papers [21] and [23] were published containing results dealing with the problem of interpretation of implication mentioned above. It is well known that Lewis has introduced a strict implication $A \rightarrow B$, which should be interpreted as ' $B$ is deducible from $A$ '. Upon this interpretation of implication some laws of the classical logic of propositions can no longer be accepted, e.g. $A \rightarrow(A \rightarrow B)$. Ackermann notices that the idea which led Lewis to the introduction of his strict implication has not been applied consistently. Thus Lewis accepts, e.g. the proposition $B \rightarrow(A \rightarrow A)$, although the correctness of $A \rightarrow A$ is independent of the correctness of $B$. Excluding such cases, Ackermann arrives at the system of so-called strong implication (strenge Implikation). This system is constructed in [21] and it is shown how modalities can be introduced into it. In [23] Ackermann shows that his system of strengthened strict implication contains a model for Lewis' system S2 of strict implication.

4. A Problem Concerning Recursive Functions. In connection with Hilbert's endeavor to give a consistent construction of the theory of real numbers, Ackermann made in 1928 in [5] an important contribution to the clarification of the notion of recursive function. He showed that there are -to use recent terminology-computable functions which are not (as we say today) primitive recursive. As an example he gives a function $f(x, y, z)$ (now called the Ackermann function) which has the property that $f(x y 0)=x+y$, $f(x y 1)=x y, f(x y 2)=x^{y}$ and for which in general the transition from $z$ to $z+1$ is an extrapolation of the transition from sums to product or from product to power. This function $f(x, y, z)$ increases so rapidly that it exceeds (for sufficiently large numbers) any primitive recursive function. This shows clearly that this function cannot itself be a primitive recursive function.

5. Investigations Concerning the Decision Problem of Predicate Logic. Since 1915 when Löwenheim wrote a paper with the title "On possibilities in the calculus of relations" (Uber Möglichkeiten im Relativkalkül) mathematical logic has been concerned with the decision problem. Algorithms 
are sought by means of which the satisfiability for classes $\mathrm{K}$ of expressions of first-order predicate logic can be decided. The classes $\mathrm{K}$ should be as comprehensive as possible. Since 1936 (Church) we know that there is no algorithm if $\mathrm{K}$ is the class of all expressions. From this it follows that there is also no such algorithm for a class $\mathrm{K}$ which is a reduction type (Reduktionstype) in such a sense that for every expression $\alpha$ of predicate logic an expression $\beta$ in $\mathrm{K}$ can effectively be found such that $\alpha$ is satisfiable exactly if $\beta$ is satisfiable. From what has been said it follows that the investigations concerning the decision problem are of two kinds: (a) indicating classes $\mathrm{K}$ for which the decision problem is solvable, (b) indicating types of reductions. Ackermann has made contributions of both kinds.

In 1928 in [6] Ackermann showed that for the class of all prenex expressions with the prefixes $\vee \ldots \vee \wedge \vee \ldots \vee$ the decision problem is solvable. This result was extended by Schütte in 1934 to the prefixes $\vee \ldots \vee \wedge \wedge \vee \ldots \vee$. In 1936 in [9] Ackermann showed the decibility of the prefixes $\wedge \vee \wedge \wedge \wedge \wedge$ if certain limiting assumptions concerning the matrix are made. Furthermore he shows here that the class of all expressions without free variables with the prefixes $\vee \wedge \vee \wedge \ldots \wedge$ is reducible in the above given sense.

Related to the decision problem is the elimination problem. Here methods are sought which allow that for certain expressions $\alpha$ of first order predicate logic the expression $\underset{p}{\vee} \alpha$ is transformed into an equivalent expression of first order predicate logic. There can be no general solution of the problem of elimination because, otherwise, predicate logic would be decidable.

In [7] Ackermann extended the problem of elimination by asking merely that corresponding to $\alpha$ there be a decidable set $\pi$ of expressions of the first order predicate logic such that $\underset{p}{\bigvee} \alpha$ is equivalent with $\Re$. He gives a solution for the case where $\alpha$ is any expression in prenex form with the prefix $\wedge \ldots \wedge$. (Here there is not always a solution for the problem of elimination in the narrower sense.)

In [8] he gives a solution for the elimination of a two placed predicate variable $P$ in the case where $\alpha$ has the form ${ }_{x} \beta$, where $x$ is not a bound variable in $\beta$ and where everywhere in $\beta P$ is followed immediately by $x$.

\section{BIBLIOGRAPHY OF WILHELM ACKERMANN}

[1] Begründung des "tertium non datur" mittels der Hilbertschen Theorie der Widerspruchsfreiheit. Mathematische Annalen, 93 (1924), 1-36.

[2] Die Widerspruchsfreiheit des Auswahlaxioms. (Vorläufige Mitteilung.) Nachrichten von der Gesselschaft der Wissenschaften zu Göttingen aus dem Jahre 1924. Mathematisch-Physikalische Klasse, 1925, 246-250.

[3] Was ist Mathematik? Zeitschrift für mathematischen und naturwissenschaftlichen Unterricht aller Schulgattungen, 58 (1927), 449-455. 
[4] D. Hilbert und W. Ackermann: Grundzilge der theoretischen Logik.

a) Springer, Berlin-Gơttingen-Heidelberg, 1st ed., 1928, VIII + 120 pp.; 2nd ed., 1938, VIII + 133 pp; 3rd ed., 1949, VIII + 155 pp; 4th ed., 1959, VIII + 188 pp.

b) Photographic reprint of the 2nd edition by Dover Publications, New York, N.Y., 1946.

c) Translations:

I) Principles of mathematical logic. English translation by Lewis M. Hammond, George G. Leckie and F. Steinhardt. Edited and with notes by Robert E. Luce. Chelsea Publishing Co。, New York, 1950, XII +172 pp.

II) Osnovy téorétičéskoj logiki. Russian translation of 2nd ed. by A. Érofeév. Edited with introductory note by S. A. Ánovskaá. Gosudarstvênnoé Izdatélstvo Innostrannoj Litératury, Moskau, 1947, $302 \mathrm{pp}$.

III) Kigô ronrigaku no kiso. Japanese translation of 3rd ed. by Makato Itô. Ôsaka Kyôiku Tosyo Kabusiki Kaisya, Tokio-Osaka, 1954, X + 207 pp.

[5] Zum Hilbertschen Aufbau der reellen Zahlen. Mathematische Annalen, 99, (1928), 118-133.

[6] Über die Erfüllbarkeit gewisser Zåhlausdrücke. Mathematische Annalen, 100 (1928), 638-649.

[7] Untersuchungen über das Eliminationsproblem der mathematischen Logik. Mathematische Annalen, 110 (1934), 390-413.

[8] Zum Eliminationsproblem der mathematischen Logik. Mathematische Annalen, 111(1935), 61-63.

[9] Beitråge zum Entscheidungsproblem der mathematischen Logik. Mathematische Annalen, 112 (1936), 419-432.

[10] Die Widerspruchsfreiheit der allgemeinen Mengenlehre. Mathematische Annalen, 114 (1937), 305-315.

[11] Mengentheoretische Begründung der Logik. Mathematische Annalen, 115 (1937), 1-22.

[12] Bemerkungen zu den logisch-mathematischen Grundlagenproblemen. In: Gonseth, F.: Philosophie mathématique. Avec cinq déclarations de MM. A. Church, W. Ackermann, A. Heyting, P. Bernays et L. Chwistek. Herman \& Chie,, Paris, 1939, 100 pp., pp. 76-82.

[13] Zur Widerspruchsfreiheit der Zahlentheorie. Mathematische Annalen, 117 (1940), 162-194.

[14] Ein System der typenfreien Logik I. Forschungen zur Logik und zur Grundlegung der exakten Wissenschaften. Neue Folge. Heft 7. S. Hirzel, Leipzig, 1941, $29 \mathrm{pp}$.

[15] Widerspruchsfreier Aufbau der Logik I. Typenfreies System ohne tertium non datur. The Journal of Symbolic Logic, 15 (1950), 33-47.

[16] Konstruktiver Aufbau eines Abschnitts der zweiten Cantorschen Zahlenklasse. Mathematische Zeitschrift, 53 (1951), 403-413.

[17] Widerspruchsfreier Aufbau einer typenfreien Logik. (Erweitertes System). Mathematische Zeitschrift, 55 (1952), 364-384. 
[18] Widerspruchsfreier Aufbau einer typenfreien Logik II. Mathematische Zeitschrift, 57 (1953), 155-166.

[19] Solvable Cases of the Decision Problem. North Holland Publishing,Co., Amsterdam, 1954, VIII + $114 \mathrm{pp}$.

[20] Zur Axiomatik der Mengenlehre. Mathematische Annalen, 131 (1956), 336-345.

[21] Begründung einer strengen Implikation. The Journal of Symbolic Logik, 21 (1956), 113-128.

[22] Philosophische Bemerkungen zur mathematischen Logik und zur mathematischen Grunlagenforschung. Ratio (Frankfurt a.M.), 1 (1957), 1-20.

English translation of [22]: Philosophical Observations on Mathematical Logik and on investigations into the foundations of mathematics. Ratio (Oxford), 1 (1957), 1-20.

[23] Über die Beziehung zwischen strikter und strenger Implikation. Dialectica, 12 (1958), 213-222.

[24] Ein typenfreies System der Logik mit ausreichender mathematischer Anwendungsfăhigkeit I. Archiv für mathematische Logik und Grundlagenforschung, 4 (1958), 3-26.

[25] Ein typenfreies System der Logik mit ausreichender mathemitischer Anwendungsfâhigkeit II. (Ableitung der Zahlentheorie). Archiv für mathematische Logik und Grundlagenforschung, 5 (1959), 96-111.

[26] Vorbemerkung und Anmerkungen. In: Nelson, L.: Beiträge zur Philosophie der Logik und Mathematik. Mit einführenden und ergänzenden Bemerkungen von W. Ackermann, P. Bernays und D. Hilbert. Verlag öffentliches Leben, Frankfurt a.M., 1959, 125 pp., p. 92 und p. 125.

[27] Grundgedanken einer typenfreien Logik. Essays on the Foundations of Mathematics. The Magnes Press. The Hebrew University, Jerusalem, 1961, 143-155.

[28] Der Aufbau einer höheren Logik. Archiv für mathematische Logik und Grundlagenforschung, 7 (1965), 5-22.

Wesfälichen Wilhelmsuniversität zu Münster

Münster in Westfalen, Germany 